\title{
THE EFFECTS OF ROTATION ON THERMAL-GRAVITATIONAL INSTABILITY IN THE PROTOGALACTIC DISK ENVIRONMENT
}

\author{
Chang Hyun Baek, ${ }^{1,2}$ Dongsu Ryu, ${ }^{3}$ Hyesung Kang, ${ }^{4}$ and Jongsoo Kim ${ }^{2}$ \\ Received 2005 December 25; accepted 2006 April 17; published 2006 May 8
}

\begin{abstract}
Thermal-gravitational instability (TGI) is studied in the protogalactic environment. We extend our previous work, in which we found that dense clumps first form out of hot background gas by thermal instability and that later a small fraction of them grow to virialized clouds of mass $M_{c} \geqslant 6 \times 10^{6} M_{\odot}$ by gravitational infall and merging. But these clouds have large angular momentum, and so they would have difficulty (although it would not be impossible) further evolving into globular clusters. In this Letter, through three-dimensional hydrodynamic simulations in a uniformly rotating frame, we explore whether or not the Coriolis force due to rotation in protogalactic disk regions can hinder binary merging and thus reduce the angular momentum of the clouds formed. With rotation comparable to the Galactic rotation at the solar circle, the Coriolis force is smaller than the pressure force during the early thermal instability stage. So the properties of the clumps formed by thermal instability are not affected noticeably by rotation, except for increased angular momentum. However, during the later stage, the Coriolis force becomes dominant over the gravity, and hence the further growth of gravitationally bound clouds by gravitational infall and merging is prohibited. Our results show that the Coriolis force effectively destroys the picture of cloud formation via TGI, rather than alleviating the problem of large angular momentum.
\end{abstract}

Subject headings: hydrodynamics — instabilities

\section{INTRODUCTION}

Thermal instability (TI; Field 1965) is often invoked to explain a variety of physical phenomena in astrophysical environments, for instance, the multiple phases of interstellar gas (e.g., Field et al. 1969; McKee \& Ostriker 1977), the formation of globular clusters (e.g., Fall \& Rees 1985; Kang et al. 2000), cooling flows in clusters of galaxies (e.g., Nulsen 1986), and the generation of turbulent flows in the interstellar medium (e.g., Vázquez-Semadeni et al. 2000; Kritsuk \& Norman 2002). Although the basic concept of TI as a local instability is rather simple and robust, the realistic situation is often more complex, involving the effects of the magnetic field, turbulence, gravity, and rotation in the case of galactic disks.

Recently, the formation of structures via thermal-gravitational instability (TGI) in the protogalactic halo environment was studied using three-dimensional numerical simulations (Baek et al. 2005, hereafter Paper I). The growth of density perturbations, initially via $\mathrm{TI}$ and subsequently via gravitational infall and merging, was followed up to 16-20 cooling times in a periodic cubic box with size $L=10 \mathrm{kpc}$. The simulations showed that clumps emerge first on scales smaller than the cooling length as a result of the nonlinear behavior of TI. Those clumps grow through compression by background pressure as well as through gravitational infall. Later, during the gravitational merging stage, some clumps become gravitationally bound, virialized clouds with mass $M_{c} \geqslant 6 \times 10^{6} M_{\odot}$ and radius $R_{c} \approx 150-200 \mathrm{pc}$. However, these massive clouds acquire angular momentum through tidal torque and merging and have a large spin parameter $\left\langle\lambda_{s}\right\rangle \sim$ 0.3 . Hence, the removal of the angular momentum from these clouds is critical, if they are to collapse further and form halo globular clusters such as in the model by Fall \& Rees (1985).

\footnotetext{
${ }^{1}$ Astrophysical Research Center for the Structure and Evolution of the Cosmos (ARCSEC), Sejong University, Seoul 143-747, Korea.

${ }^{2}$ Korea Astronomy and Space Science Institute, Daejeon 305-348, Korea.

${ }^{3}$ Department of Astronomy and Space Science, Chungnam National University, Daejeon 305-764, Korea.

${ }^{4}$ Department of Earth Sciences, Pusan National University, Pusan 609-735, Korea.
}

In this Letter we study the effects of rotation in protogalactic disk regions on the formation of clouds via TGI. Uniform rotation was included in the same numerical simulations as in Paper I to model the protogalactic disk environment. In the next section we describe our models and numerical method. The simulation results are presented in $\S 3$. A summary follows in $\S 4$.

\section{NUMERICAL SIMULATIONS}

As in Paper I, we consider a primordial gas of $T_{h}=1.7 \times$ $10^{6} \mathrm{~K}$, which corresponds to the canonical temperature of an isothermal sphere with circular velocity $V_{c}=220 \mathrm{~km} \mathrm{~s}^{-1}$, representing the hot phase of the gas in disk galaxies like the Milky Way. The fiducial value of the mean background density of hydrogen nuclei was chosen to be $n_{h}=0.1 \mathrm{~cm}^{-3}$. For the primordial gas with an assumed ratio of $\mathrm{He} / \mathrm{H}$ number densities of $1 / 10$, the gas mass density is given by $\rho_{h}=\left(2.34 \times 10^{-24} \mathrm{~g}\right) n_{h}$. With $T_{h}=1.7 \times 10^{6} \mathrm{~K}$ and $n_{h}=0.1 \mathrm{~cm}^{-3}$, the initial cooling timescale is $t_{\text {cool }}=2 \times 10^{7} \mathrm{yr}$. On the other hand, the free-fall timescale, or the gravitational timescale, is $t_{\text {grav }}=1.4 \times 10^{8} \mathrm{yr} \approx$ $7 t_{\text {cool }}$. Note that $t_{\text {cool }} \propto n_{h}^{-1}$, while $t_{\text {grav }} \propto n_{h}^{-1 / 2}$. So cooling, compared to gravitational processes, becomes relatively more important at higher densities and vice versa. The cooling length scale is defined as $l_{\text {cool }}=c_{h} t_{\text {cool }}=4 \mathrm{kpc}$, where $c_{h}=198 \mathrm{~km} \mathrm{~s}^{-1}$ is the sound speed.

To model the protogalactic disk environment, uniform rotation was incorporated by using a Cartesian coordinate system that rotates with angular velocity, $\Omega \hat{z}$. We chose as a fiducial value for the angular speed $\Omega_{0}=27 \mathrm{~km} \mathrm{~s}^{-1} \mathrm{kpc}^{-1}$, which corresponds to the Galactic rotation at the solar circle $\left(R_{0} \approx\right.$ $8.5 \mathrm{kpc}$; Feast \& Whitelock 1997). A case with lower angular speed, $\Omega_{0} / 2$, was also considered for comparison. In the rotating frame, an additional Coriolis force term, $f_{c}=-2 \Omega \hat{z} \times \boldsymbol{v}$, is added to the equation of motion. The simulation box was set to be cubic, with size $L=10 \mathrm{kpc}=2.5 l_{\text {cool }}$. The size was chosen to be large enough to accommodate a fair number of thermally unstable clouds of cooling length size and therefore to obtain good statistics of cloud properties. A periodic bound- 
TABLE 1

Model Parameters for Simulations

\begin{tabular}{cccc}
\hline \hline Model & Grid Zones & $t_{\text {end }}\left(t_{\text {cool }}\right)^{\mathrm{a}}$ & $\Omega^{\mathrm{b}}$ \\
\hline S1024 $\ldots \ldots .$. & $1024^{3}$ & 16 & 0.0 \\
R1024 $\ldots \ldots$. & $1024^{3}$ & 16 & $\Omega_{0}$ \\
RH512 $\ldots \ldots$. & $512^{3}$ & 20 & $0.5 \Omega_{0}$ \\
\hline${ }^{\mathrm{a}} t_{\text {cool }}=2 \times 10^{7} \mathrm{yr}$ & & \\
${ }^{\mathrm{b}} \Omega_{0}=27 \mathrm{~km} \mathrm{~s}^{-1} \mathrm{kpc}^{-1}$. & &
\end{tabular}

ary condition was imposed on the box, although it might not be the most natural choice for a disklike geometry. We believe this particular choice of boundary condition would not affect the main conclusion regarding the role of rotation. Our periodic, cubic, simulation box represents a volume of the disk region with significant rotation inside a protogalaxy.

To mimic the density perturbations that existed on a wide range of length scales inside the protogalaxies, the initial density field was drawn from random Gaussian fluctuations with a predefined density power spectrum. The density power spectrum was assumed to be given by $P_{k} \propto k^{n}$ with $n=0$ (white noise). In Paper I we showed that the properties of clouds, once formed, do not depend on the initial fluctuation spectrum, although their spatial distribution is sensitive to the spectrum. Without a priori knowledge of the initial fluctuations, a spectrum of constant power over all scales was chosen. The amplitude of the density power spectrum was fixed by the condition $\delta_{\mathrm{rms}} \equiv\left\langle\delta \rho^{2}\right\rangle^{1 / 2} /\langle\rho\rangle=0.2$. The initial temperature was set to be uniform, and the initial velocity was set to be zero everywhere.

The evolution of the gas from the initial perturbations was followed with (1) radiative cooling due to the primordial gas (Sutherland \& Dopita 1993) down to $T=10^{4} \mathrm{~K}$, (2) background heating equal to the cooling of the initial, unperturbed background gas, and (3) self-gravity. The hydrodynamics was solved using an Eulerian hydrodynamic code based on the total variation diminishing scheme (Ryu et al. 1993) on a grid with $1024^{3}$ cells (or $512^{3}$ cells in the comparison run with $\Omega_{0} / 2$ ). Simulations started at $t=0$ and lasted up to $t_{\text {end }}=(16-20) t_{\text {cool }}$. Three simulations are presented in this Letter, differing in angular speed. Model parameters are summarized in Table 1.

\section{RESULTS}

We begin our discussion by comparing the Coriolis force to the pressure force and gravity for the flows associated with the formation of clouds. First, the ratio of the Coriolis force to the pressure force can be estimated roughly as

$$
\frac{f_{c}}{f_{p}} \sim \frac{2 \Omega_{0} v}{P_{h} / R_{c} \rho_{h}} \sim \frac{10 \Omega_{0} R_{c}}{3 c_{h}},
$$

where the typical flow speed is assumed to be similar to the sound speed, i.e., $v \sim c_{h}$, and the adiabatic index of gas is $\gamma=5 / 3$. Here $R_{c}$ is the radius of typical clouds. Conservatively with $R_{c} \sim 100 \mathrm{pc}$, the ratio is $f_{c} / f_{p} \sim 0.05$. So we expect that the effects of rotation are small, if not negligible, during the TI stage. The ratio of the Coriolis force to gravity can be written roughly as

$$
\frac{f_{c}}{f_{g}} \sim \frac{2 \Omega_{0} v R_{c}^{2}}{G M_{c}} .
$$

Again with $v \sim c_{h}$, and conservatively with $M_{c} \sim 10^{6} M_{\odot}$ and $R_{c} \sim 100 \mathrm{pc}$, the ratio becomes $f_{c} / f_{g} \sim 25$. Hence, during the gravitational infall and merging stages, the Coriolis force is expected to play a dominant dynamical role in our simulations with rotation.

The Coriolis force causes flows to be deflected at the right angle to the flow direction; hence, it hinders spherical infall motions toward high-density peaks and generates circular motions in the plane perpendicular to the rotation axis. The Coriolis force also hinders the merger of two clumps by causing them to drift in opposite directions perpendicular to the line connecting the two clouds, and this leads to stretched wormlike structures. As a result, the formation of knotlike structures is suppressed, and instead filamentary and sheetlike structures seem to appear.

Figure 1 shows the density power spectrum at different times. In the figure, the dimensionless wavenumber is given as $k \equiv$ $L / \lambda$. The power spectrum is presented in a way that $\int P_{k} d k=$ $\left\langle\rho^{2}\right\rangle$. During the early stage, $t \leqslant 6 t_{\text {cool }}$, the evolution of the density power spectrum looks similar in the two models S1024 and R1024. This is because, with $t_{\text {cool }}<t_{\text {grav }}$, initially the power grows mostly due to TI, and the Coriolis force has negligible effects. During $t \geqslant 6 t_{\text {cool }}$, gravity controls the growth, and so the two models evolve differently. In model S1024, the power continues to grow due to gravitational infall and merging over all scales. In model R1024, on the other hand, the Coriolis force suppresses the growth, more so, evidently, on smaller scales. The comparison of the power spectrum of model R1024 $\left(\Omega=\Omega_{0}\right)$ with that of model RH512 $\left(\Omega=0.5 \Omega_{0}\right)$ demonstrates the effects of the

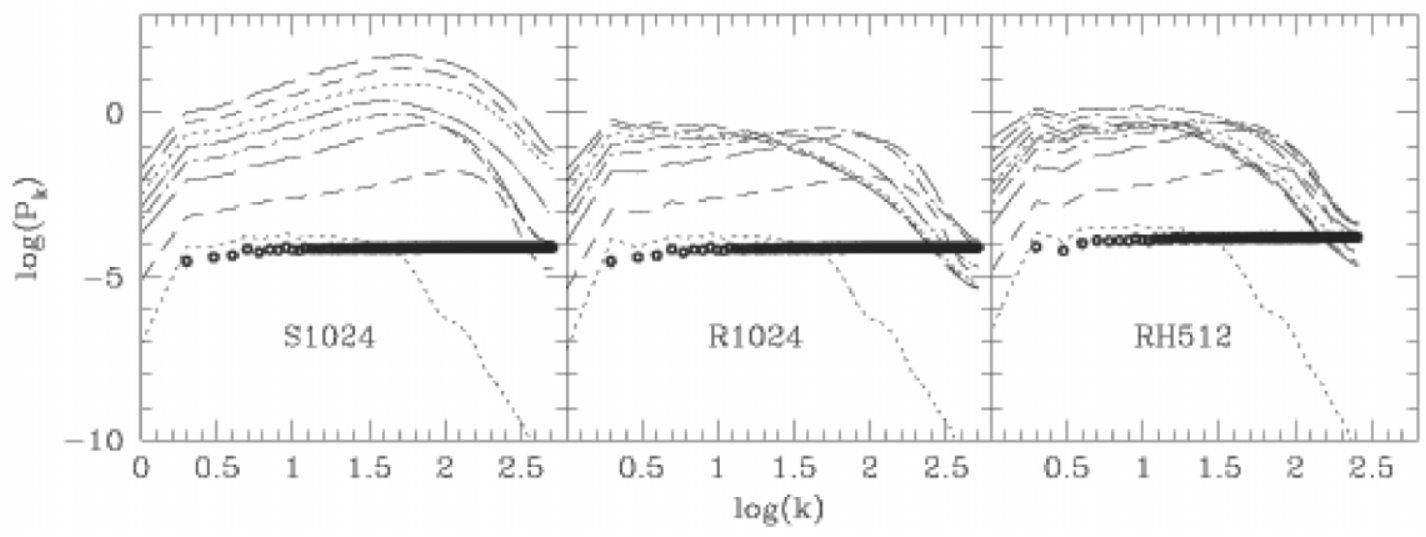

FIG. 1.-Evolution of the density power spectrum in models S1024, R1024, and RH512. Circles represent the initial power spectrum at $t=0$. Lines show the power spectrum at $2 t_{\text {cool }}, 4 t_{\text {cool }}, 6 t_{\text {cool }}, \ldots, 16 t_{\text {cool }}$ in models S1024 and R1024, and at $4 t_{\text {cool }}, 6 t_{\text {cool }}, \ldots, 20 t_{\text {cool }}$ in model RH512. 


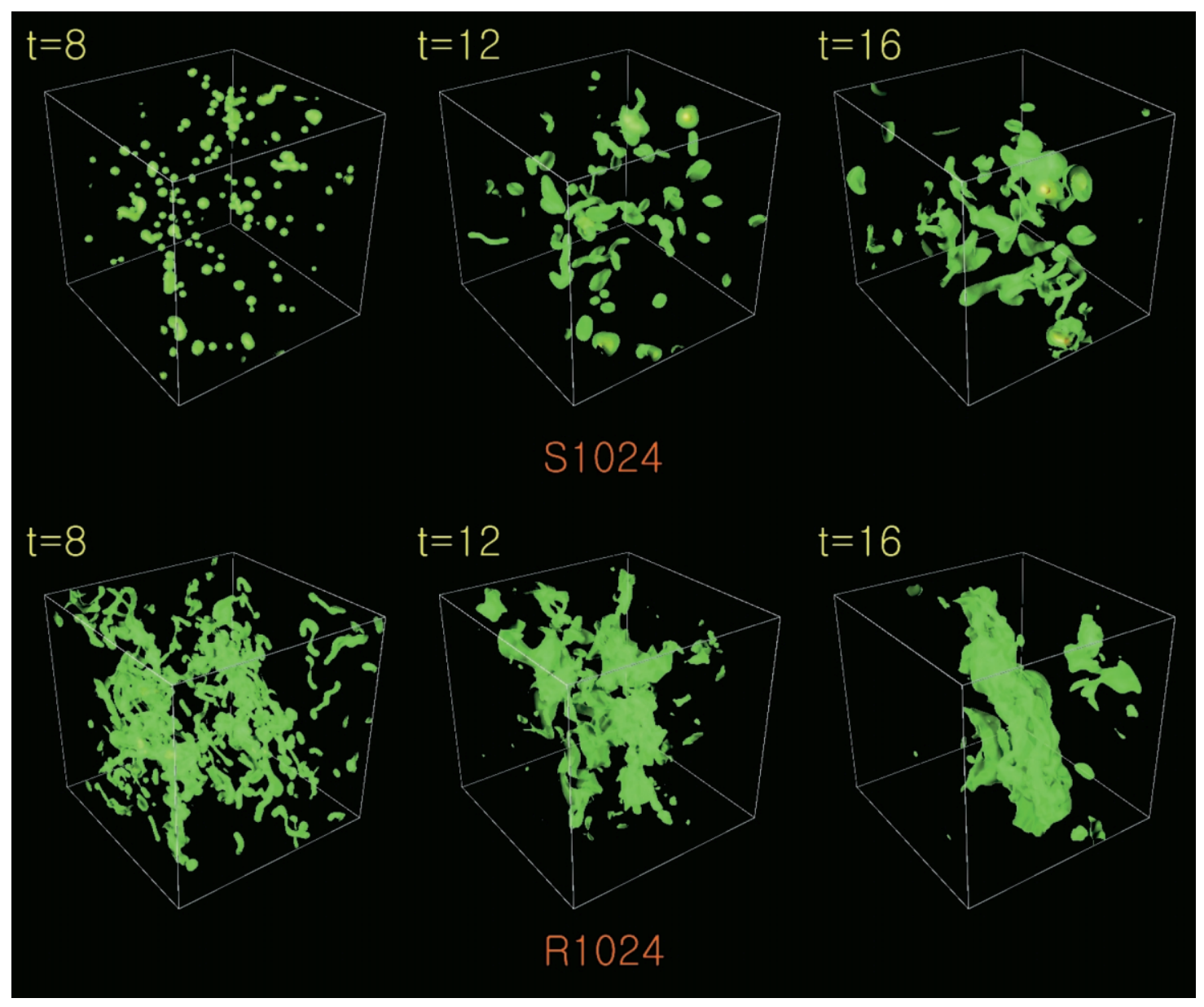

FIG. 2.-Isodensity surfaces inside a $2.5 \mathrm{kpc}$ box of $256^{3}$ grid zones, which is $(1 / 4)^{3}$ of the total simulation box, at $8 t_{\text {cool }}, 12 t_{\text {cool }}$, and $16 t_{\text {cool }}$ in models $S 1024$ (top) and R1024 (bottom). Green surfaces correspond to $10 \rho_{0}$, yellow surfaces to $10^{2} \rho_{0}$, and red surfaces to $10^{3} \rho_{0}$. Here $\rho_{0}$ is the mean initial density. The box is oriented in such a way that the $x$-, $y$-, and $z$-axes are from near to far, from bottom to top, and from left to right, respectively. Rotation is along the $z$-axes in model R1024.

different rotation speeds. The figure shows that even with a halfrotation speed, the Coriolis force suppresses the growth of the power spectrum effectively.

To look at the shape and spatial distribution of formed structures, three-dimensional isodensity surfaces of a cubic region are shown in Figure 2. Initially clumps appear via TI in both models, as discussed in Paper I (see their Fig. 3). In model S1024, by $t=8 t_{\text {cool }}$ the clumps have developed into distinct clouds with roughly spherical shapes. By $t=12 t_{\text {cool }}$ some clouds have grown to be massive enough to become gravitationally bound, and by $t=16 t_{\text {cool }}$ they have grown more massive. Those gravitationally bound clouds have a central density higher than 1000 times the mean background density. In model R1024, on the other hand, the initial clumps do not grow to distinct clouds. Instead, loosely connected filamentary and sheetlike structures appear with a central density lower than 100 times the mean background density.

Although clouds are not distinctively defined in model R1024, we still identified clouds around high-density peaks using the algorithm clumpfind described in Paper I and calculated their properties. Starting from the top, the first row of Figure 3 shows the number of identified clouds, $N_{c}$, as a function of their mass, $M_{c}$. The mass function of the two models is almost identical at $t=4 t_{\text {cool }}$. It is roughly Gaussian, since the initial density perturbations were drawn from a random
Gaussian distribution. In model S1024, the mass function has evolved roughly into a lognormal distribution by $t=8 t_{\text {cool }}$, which is a signature of nonlinear structure formation. At the later stage, the mass function extends to a higher mass with a power-law distribution, as more massive clouds develop through gravitational merging. In model R1024, however, the mass distribution remains roughly Gaussian at $t=8 t_{\text {cool }}$, and later it develops into a form that is not well defined. This is another indication that the development of nonlinear structures has been severely altered by the Coriolis force.

The second row of Figure 3 shows the energy ratio of identified clouds, $\beta=2\left(E_{K}+E_{T}\right) /\left|E_{G}\right|$. Here $E_{K}$ is the kinetic energy defined in the center of mass of a given cloud, $E_{T}$ is the thermal energy, and $E_{G}$ is the gravitational energy. The parameter $\beta$ tells us whether or not clouds are gravitationally bound $(\beta \lessgtr 2)$, and whether or not they are in virial equilibrium $(\beta \sim 1)$. The figure shows that the virialized clouds with $M_{c} \geqslant 6 \times 10^{6} M_{\odot}$ have formed in model S1024. But in model R1024 the identified objects have $M_{c} \lesssim 10^{6} M_{\odot}$, and none are gravitationally bound. We note that even in model RH512 with a half-rotation speed, no gravitationally bound clouds were found.

The bottom row of Figure 3 shows the specific angular momentum of identified clouds. At $t=4 t_{\text {cool }}$ the specific angular momentum is different in the two models, although other properties are similar. As mentioned earlier, clumps formed in the 


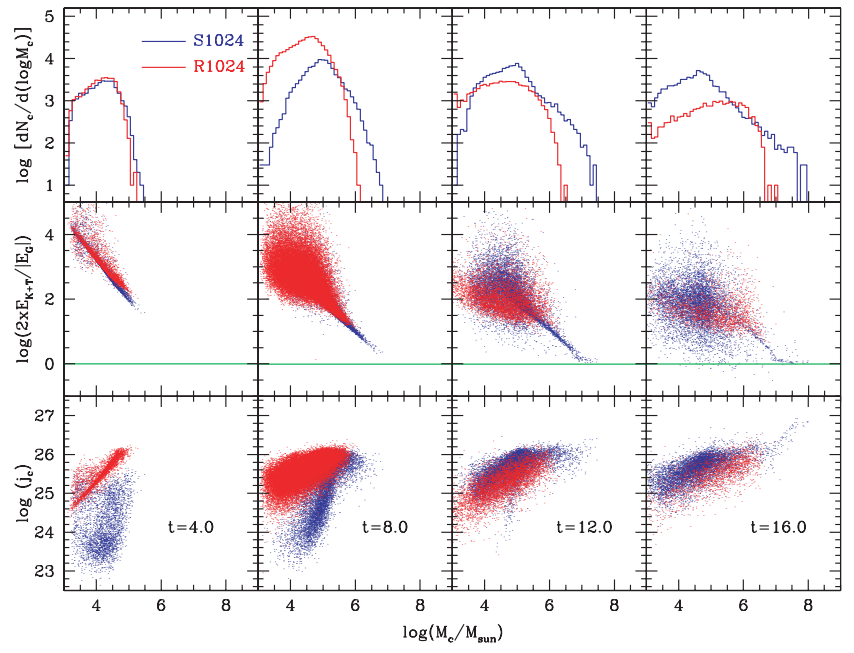

FIG. 3.-Differential number of clouds $d N_{c} / d\left(\log M_{c}\right)$, energy ratio $2\left(E_{T}+E_{K}\right) /\left|E_{G}\right|$, and specific angular momentum $j_{c}$, as a function of cloud mass $M_{c}$, at four different times in models S1024 (blue color) and R1024 (red color). Here $j_{c}$ is in cgs units.

early stage gain angular momentum through the Coriolis effect, so $j_{c}$ is higher in model R1024 than in model S1024, but during the later stage, angular momentum is acquired efficiently through torquing and merging in model S1024; this is not the case for model R1024. Hence, for a given mass, $j_{c}$ becomes higher in model S1024 than in model R1024.

\section{SUMMARY}

We study the effects of rotation in protogalactic disk regions on the formation of structures via TGI in the protogalactic environment. A simplified setting was considered, where a gas of primordial composition evolves from initial density perturbations in a uniformly rotating box. In Paper I, we found that without rotation, virialized clouds of mass $M_{c} \gtrsim 6 \times 10^{6} M_{\odot}$ can form as a result of TGI, although they form with large angular momentum of spin parameter $\left\langle\lambda_{s}\right\rangle \sim 0.3$. In this Letter we find that with rotation with an angular speed comparable to that of the Galactic rotation at the solar circle, $\Omega_{0}=27 \mathrm{~km} \mathrm{~s}^{-1} \mathrm{kpc}^{-1}$, the Coriolis force suppresses gravitational infall and merging and disperses the gas to filamentary and sheetlike structures. As a result, instead of massive virialized clouds formed in nonrotating models, clumps with $M_{c} \lesssim 10^{6} M_{\odot}$, which are gravitationally unbound and often transient, are only found.

We conclude that, rather than alleviating the angular momentum problem discussed in Paper I, rotation in protogalactic disk regions has a destructive effect on the formation of clouds. The results in this Letter and those in Paper I combined imply that it would be difficult for globular clusters to have formed via TGI in protogalaxies, and even less likely in rotating disk regions.

A few notes on our results: (1) Primordial gas is considered in this Letter, but adding metal of order of $0.1 Z_{\odot}$, which would enhance thermal processes, does not change the results, because it is the gravitational processes that are responsible for the formation of massive clouds but are suppressed by the Coriolis force. We confirmed this with another simulation with metallicity of $0.1 Z_{\odot}$, although we do not report the simulation in this Letter. We point out, however, that changing other parameters, for instance increasing the gas density by a factor of 10 , would make a difference. (2) The size of the simulation box that we used, $L=10 \mathrm{kpc}$, along with the periodic boundaries, is too large to represent a protogalactic disk region. In order to check the effects of box size, we performed simulations in a smaller box of $2.5 \mathrm{kpc}$ with $256^{3}$ cells with and without rotation. Again, although we do not report the simulation in detail, the results confirmed that the formation of gravitationally bound objects is prohibited by the Coriolis force, regardless of the box size. (3) To be more realistic, differential rotation, instead of uniform rotation, needs to be investigated.

We thank the anonymous referee for clarifying comments. The work of C. H. B., H. K., and J. K. was supported by KOSEF through ARCSEC. The work of D. R. was supported by a Korea Research Foundation grant (KRF-2004-015-C00213). Simulations were run using Linux clusters at the KASI and KISTI Supercomputing Center.

\section{REFERENCES}

Baek, C. H., Kang, H., Kim, J., \& Ryu, D. 2005, ApJ, 630, 689 (Paper I)

Fall, S. M., \& Rees, M. J. 1985, ApJ, 298, 18

Feast, M., \& Whitelock, P. 1997, MNRAS, 291, 683

Field, G. B. 1965, ApJ, 142, 531

Field, G. B., Goldsmith, D. W., \& Habing, H. J. 1969, ApJ, 155, L149

Kang, H., Lake, G., \& Ryu, D. 2000, J. Korean Astron. Soc., 33, 111
Kritsuk, A. G., \& Norman, M. L. 2002, ApJ, 569, L127

McKee, C. F., \& Ostriker, J. P. 1977, ApJ, 218, 148

Nulsen, P. E. J. 1986, MNRAS, 221, 377

Ryu, D., Ostriker, J. P., Kang, H., \& Cen, R. 1993, ApJ, 414, 1

Sutherland, R. S., \& Dopita, M. A. 1993, ApJS, 88, 253

Vázquez-Semadeni, E., Gazol, A., \& Scalo, J. 2000, ApJ, 540, 271 